\title{
Abordaje clínico de la dependencia a nicotina desde un enfoque sistémico-relacional. Resultados de un estudio descriptivo de serie de casos.
}

\author{
José María Fuentes-Pila'; Esther lópez García²; Pedro Calatayud ${ }^{3}$; Roberto Pereira ${ }^{4}$ \\ (1) Instituto Cántabro de Conductas Adictivas (ICCA). Director. \\ (2) ICCA. \\ (3) ICCA. Director Médico \\ (4) Psiquiatra. Escuela Vasco Navarra de Terapia Familiar. Director.
}

Enviar correspondencia a:

JM Fuentes-Pila. Instituto Cántabro de Conductas Adictivas. C/ Arrabal, 25. 39003 Santander. Tel.: 942 223106. e-mail: jmfarmacia@sistelcom.com

Recibido: Agosto 2005

Aceptado: Diciembre 2006

\section{RESUMEN}

Se presenta en este trabajo un estudio descriptivo de serie de casos en tratamiento de adicción a nicotina mediante programa multicomponente, aplicando un enfoque terapéutico sistémico- relacional. Mientras que un buen número de fumadores consiguen la abstinencia a través de los diferentes programas con distintos niveles de complejidad en función de su dependencia, hay un grupo de fumadores que asocian sus dificultades para la cesación tabáquica con aspectos relacionados con su situación de ciclo vital, problemas con su familia de origen o con sus redes sociales. Se revisan los aspectos clínicos más relevantes del modelo y se presentan los resultados de un estudio descriptivo sobre una serie de 128 pacientes, de los cuales el 60,50\% expresaron problemas familiares y/o personales que dificultaban sus posibilidades de éxito en el programa.

Tras una intervención de un año de duración en la que se aplican 25 sesiones de terapia junto con terapia sustitutiva con nicotina combinada durante los 3 primeros meses de tratamiento, se mantuvieron abstinentes el 77,3\%.

En la actualidad se ha profundizado de manera muy apreciable en el conocimiento de la adicción a nicotina, dándose respuestas distintas a las diferentes demandas asistenciales, así como a los diferentes niveles de complejidad que presente el cuadro adictivo. Consideramos que las aportaciones del modelo terapéutico sistémico - relacional en sus diferentes formas de entender la intervención, pueden mejorar los resultados de tratamiento. Aumentar el grado de complejidad de las intervenciones se justifica cuando existen complicaciones en el abordaje clínico de la adicción a nicotina mediante otros modelos de tratamiento de menor intensidad terapéutica.

Palabras clave: dependencia a nicotina, enfoque sistémico relacional, terapia sustitutiva con nicotina.

\section{SUMMARY}

We present in this work a descriptive study of series of cases in treatment for nicotine addiction with a multicomponent program focusing on a systemic-relational therapy. While a good number of smokers are able to stop smoking, with the help of different programs with different levels of complexity depending on their level of addiction, there is a group of smokers who associate their difficulty to stop smoking with aspects related to their life cycle situation, problems with their family of origin or with their social network. We revised the most relevant clinical aspects of the model and presented the results of a descriptive study of a series of 128 patients, of wich $60,50 \%$ expressed family and/or personal problems that made the possibilities of success in the program more difficult.

After an intervention that lasted a year during wich there were 25 therapy sessions together with combined substitute therapy with nicotine (STN) during the first 3 months of treatment, $77,30 \%$ maintained abstinence.

Nowadays we know much more about nicotine addictions, giving different answers to different needs, just like the different levels of complexity of each addict. We consider that the contributions of the systemic- relational model in its different ways of understanding the intervention, can better the results of the treatment.

We are justified in increasing the level of complexity of the interventions when there exist complications in the clinical handling of nicotine addiction because of other models of treatment of lesser intensity.

Key words: nicotine addiction, systemic-relational model, substitution therapy of nicotine (STN). 


\section{INTRODUCCIÓN}

Los tratamientos existentes para el tratamiento de la dependencia a nicotina, en sus diferentes modalidades y niveles de complejidad en las intervenciones parece ser útil para un buen número de fumadores. El control del tabaquismo es, en la actualidad, el reto más difícil con el que se enfrentan los gobiernos de los países desarrollados en términos de morbi-mortalidad. Y es, posiblemente ésta, una de las razones por las que no se esté tratando otra cosa que "el fumar" de las personas, alejándonos del análisis de la relación "tabaco-sujeto-contexto-tabaco", aspecto, en algunos casos relevante para entender las dificultades abstinenciales a largo plazo de los fumadores. En la actualidad, 1.100 millones de personas fuman en todo el mundo. Se prevé que en el año 2.025, sean 1.600 millones las personas fumadoras ${ }^{1}$. Otro motivo de alarma sanitaria es la constatación del impacto del consumo de tabaco sobre la población de no fumadores. En el año 2.000 fallecieron en el mundo 4,8 millones de personas, incidiendo la mortalidad de manera semejante en países desarrollados y en países en vías de desarrollo² . España presentaba una carga de mortalidad de 55.613 fallecimientos atribuibles al consumo de tabaco en 19983. En 2001, 49.072 personas murieron en España por enfermedades debidas al consumo de tabaco ${ }^{4}$, lo cual hace pensar con cierto optimismo en el conjunto de acciones preventivas y restrictivas vertebradas en el Plan Nacional para la Prevención y Control del Tabaquismo aprobado en 2003.

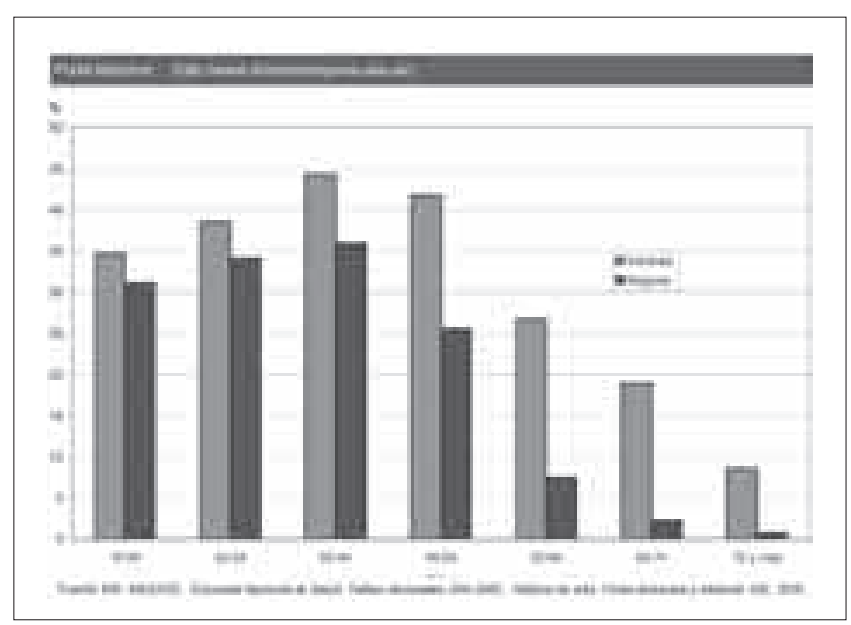

FIGURA 1

Población que fuma diariamente según sexo y edad, 2003

Los datos para España han mejorado significativamente en los últimos 5 años. Mientras que la Encuesta Nacional de Salud de $2001^{5}$ indicaba una prevalencia de consumo de tabaco en población de 16 años o más del 34,4\%, la realizada en 2003, muestra una prevalencia inferior al 30\%. Concretamente, del $28,1 \%{ }^{6}$. Parece, pues, que la lenta reducción que se venía produciendo desde 19897, ha emprendido un ritmo esperanzador en la lucha contra el tabaquismo.

\section{La dependencia a nicotina}

La dependencia a nicotina está reconocida como trastorno mental y de comportamiento en la Clasificación Internacional de Enfermedades de la $\mathrm{OMS}^{8}$ y en el Manual de Diagnóstico y Estadístico de los Trastornos Mentales (DSM-IV-TR) ${ }^{9}$ bajo de denominación de Trastorno de dependencia a nicotina. En este trabajo, nos permitimos proponer una ampliación de la denominación de esta patología adictiva. Dependencia nicotín-tabáquica, término no admitido en castellano, ni en las clasificaciones internacionales sobre trastornos adictivos, pero que, desde nuestro punto de vista permite expresar la complejidad de la conducta adictiva asociada al cigarrillo, tanto desde el punto de vista neurobiológico (nicotina), como desde el plano emocional, relacional y conductual (tabaco).Un paso más a los términos dependencia a nicotina y adicción a nicotina ${ }^{10}$, que dejamos abierto al debate.

Fumar genera una intensa compulsión, como consecuencia de la elevada capacidad adictiva de la nicotina ${ }^{11,12}$. Resulta, pues, razonable, evitar una terminología cuanto menos equívoca, ampliamente utilizada en diferentes medios de comunicación, como lo es hablar "del hábito de fumar"13. La conducta de consumo se incorpora en la vida del sujeto en su primera adolescencia, para posteriormente integrarse a los diferentes planos de la vida emocional, afectiva, relacional, cognitiva y conductual de la persona. Esto hace que los fumadores tengan muchas dificultades para proyectar su propia auto- imagen como no fumadores.

La autoimagen, entendida como autoconcepto, es uno de los tres componentes esenciales del self o personalidad integral ${ }^{14}$. La autoimagen o autoconcepto, se entiende como la imagen que uno tiene de sí mismo y que se encuentra determinada por la acumulación de la información tanto interna como externa, juzgada y valorada mediante la interacción de sistemas de estilos (forma específica que tiene el individuo de razonar sobre la información) y valores (selección de aspectos significativos de dicha información ${ }^{15}$ ). La importancia de la capacidad para analizarse ha sido destacada por Gardner ${ }^{16}$, dentro de la inteligencia que el autor ha denominado intrapersonal. En el campo de la instrucción, Gallagher ${ }^{17}$, destaca el autoconcepto como una de las variables más relevantes dentro de los nuevos modelos de enseñanza y aprendizaje. También contribuye a la complejidad de la dependencia a nicotina la forma de consumo, su accesibilidad en el mercado y los bajos precios del producto ${ }^{18}$.

La adolescencia es, por lo general, la etapa en la que comienza el consumo de tabaco. El inicio del intento de desvinculación de la familia de origen, así 
como la configuración de la esfera relacional externa (búsqueda de apoyo y aprobación), se acompañan de toda una serie de rituales de identificación grupal, que pueden verse facilitados por la presencia de los mismos en el seno de la familia de origen ${ }^{19}$. El tabaco, como consecuencia de la amable representación social que ha tenido hasta hace menos de 5 años, aparece como un poderoso elemento de identificación entre pares, para instalarse de manera definitiva en una buena parte de estos jóvenes.

Resulta interesante constatar la, todavía, elevada prevalencia del tabaquismo en nuestro país, en contraposición con el deseo de dejar de fumar de la mayoría de los fumadores activos. De hecho, el 60\% de los fumadores han intentado dejar de fumar en alguna ocasión ${ }^{20}$. Hasta hace 15 años, las medidas adoptadas en el control del tabaquismo eran escasas o nulas y, la mayoría de los fumadores que dejaron de fumar, lo hicieron sin ningún tipo de asistencia ${ }^{21}$.

Sin embargo, solo el $2 \%$ al año de los fumadores que realizan intentos de cesación espontáneos (sin ningún tipo de ayuda) tienen éxito ${ }^{20}$.

\section{Aspectos generales del abordaje clínico de la dependencia a nicotina}

A pesar de la inclusión de los Trastornos relacionados con la nicotina en los Manuales Diagnósticos, en la práctica clínica son pocos los dispositivos que aplican estos criterios como elementos de base para la exploración en profundidad de la relación entre sujeto y sustancia adictiva. Numerosas asociaciones implicadas en el tratamiento del tabaquismo han realizado un documento de consenso ${ }^{22}$ en el que el "reduccionismo" es el denominador común, con el fin, posiblemente, de llegar al mayor número posible de intervinientes que ayuden al mayor número posible de fumadores. Se ha avanzado significativamente en el diagnóstico de la dependencia a nicotina, a través de variadas herramientas, si bien siguen sin ser de uso extendido entre los clínicos: Los autorregistros ${ }^{23}$, así como la evaluación del craving ${ }^{24,25}$ como síntoma abstinencial prioritario en la gestión de una abstinencia duradera o las escalas de autoeficacia ${ }^{26,27}$ y de expectativas de resultados ${ }^{28}$ aumentan la fiabilidad diagnóstica de la dependencia a nicotina, posibilitando la adecuada planificación de los programas, así como de las herramientas terapéuticas a utilizar, dependiendo del perfil adictivo de cada paciente.

El Cuestionario de Tolerancia de Fagerström ${ }^{29,} 30$, abrió el camino de la evaluación de la adicción a nicotina de manera específica y operativa desde el punto de vista clínico. El Test de Fagerström De Dependencia a Nicotina Modificado (TFDNM), su versión actualizada ${ }^{31}$, sigue siendo la herramienta diagnóstica más ampliamente utilizada en la práctica clínica diaria utili- zada no solo para el diagnóstico, sino para la definición del tipo de terapia farmacológica más adecuada ${ }^{32,33}$.

En la actualidad podemos considerar que son tres los pilares sobre los que se soporta la intervención especializada en la dependencia a nicotina: El Modelo Transteórico de Cambio 34,35 (MTC), las intervenciones farmacológicas y la Prevención de Recaídas.

EI MTC, se ha manejado más como un rudimento diagnóstico de las posibilidades de interacción con un paciente, que como un encuadre que permita entender resistencias, creencias, motivación para el cambio y recursos del fumador. La utilidad clínica de este modelo, reside en el análisis de los "niveles de cambio" y de los "procesos de cambio", que aumentan el grado de complejidad a la vez que enriquecen las posibilidades de interacción psicoterapéutica. Los niveles de cambio indican la posibilidad de actuar en diferentes planos de la demanda (síntoma, conflictos interpersonales, cogniciones desadaptativas, conflictos familia /sistema ${ }^{35}$ ) .

Los clínicos, desde nuestro punto de vista, deberíamos manejar el MTC con el objeto de conocer mejor las motivaciones para el cambio de las personas en su relación con la sustancia adictiva ${ }^{36,37}$.

El objetivo fundamental de cualquier programa dirigido a la abstinencia, es el mantenimiento de la misma a largo plazo. La recaída es, no solo parte del proceso de tratamiento, sino el principal problema con el que se encuentran los terapeutas que trabajan en adicciones.

El Modelo de prevención de Recaídas desarrollado por Marlatt y Gordon ${ }^{38}$, es un constructo que tiene su base en la Teoría del Aprendizaje social de Bandura ${ }^{39}$, y que cobra importancia clínica a partir de los años 90. Su objetivo principal, dar explicación al fenómeno del desliz y de la recaída, diferenciándolos, así como dando explicación y respuestas a las dificultades que presentan los pacientes.

No siendo motivo de este trabajo una revisión en profundidad de las diferentes modalidades terapéuticas de tratamiento del tabaquismo, cabe destacar que la importancia del uso de fármacos en el abordaje clínico de la dependencia a nicotina, ha ido en aumento en los últimos 20 años. Así como el modelo biologicista farmacológico ha ocupado gran parte de la atención a la Salud Mental, incluidas las drogodependencias, de igual manera está ocurriendo en la atención a los fumadores. El interés de las compañías farmacéuticas por un mercado potencialmente muy importante facilita el desarrollo de nuevos fármacos para "dejar de fumar".

A las ya clásicas herramientas farmacológicas (Terapia Sustitutiva con Nicotina-TSN- y bupropion) que han demostrado su eficacia en el tratamiento de los fumadores ${ }^{40}$, se unirán otros fármacos como 
el Rimonabat, bloqueante de los receptores cannabinoides de CB-1 o el Varenicline, agonista parcial de receptores nicotínicos ${ }^{41}$. Incluso la naltrexona, parece haber mostrado eficacia en el tratamiento de mujeres fumadoras ${ }^{42}$

\section{Eficacia de las diferentes formas de tratamiento de la dependencia nicotín-tabáquica}

Son muchos y muy variados los tipos de abordaje clínico del tabaquismo. Y muy numerosas las revisiones de los distintos tipos de tratamiento. La revisión más amplia sobre la eficacia de los diferentes procedimientos para dejar de fumar es la de Schwartz ${ }^{43}$, que aunque tiene ya más de 15 años sigue dando concordancia con los datos de nuevas revisiones.

A partir de un $20 \%$ de abstinencia al año se considera que un programa para dejar de fumar "funciona", ya que duplica o triplica el nivel obtenido por el grupo control ${ }^{44}$. Según la revisión anteriormente mencionada, los 10 procedimientos más eficaces para dejar de fumar serían los siguientes, indicándose entre paréntesis, el porcentaje de los ensayos con tasas mayores del 33\% (límite por debajo del cual, la revisión no los considera eficaces, aumentando en un $13 \%$ el nivel de exigencia) a los 12 meses de seguimiento:

1)Programas psicológicos multicomponentes (65\%);2)Intervención médica con pacientes con problemas cardiacos (63\%);3)Saciación de fumar con otros procedimientos (58\%); 4)Intervención médica con pacientes que presentan problemas pulmonares (50\%); 5)Fumar rápido combinado con otros procedimientos (50\%); 6) Reducción gradual de la ingesta de nicotina y alquitrán (44\%); 7) Prevención de factores de riesgo (43\%); 8) Programas con formato grupal $(39 \%)$; 9) Hipnosis individual $(38 \%)$; 10)Chicle de nicotina y tratamiento conductual (38\%).

Los porcentajes de eficacia media oscilan entre el $40 \%$ de los programas multicomponentes, al 19\% de la hipnosis individual.

Posteriores revisiones de la literatura realizados por especialistas internacionales en el tratamiento del tabaquismo ${ }^{45,46,47}$ permiten concluir que una de las estrategias más eficaces para dejar de fumar en tratamientos especializados son los programas psicológicos multicomponentes (tratamiento psicológico que une dos o más técnicas orientadas al cambio de conducta) con base cognitivo-conductual, pudiendo incrementarse su eficacia con procedimientos farmacológicos (chicles o parches de nicotina), siendo prácticamente coincidentes con la revisión anteriormente mencionada de Schwartz. Focalizando la atención en tratamientos psicológicos, en algunas de las revisiones en las que se estudia la eficacia de la intervención representada por los odds ratio (OR) entre el grupo de tratamiento y el grupo control con un intervalo de confianza del 95\%, la eficacia de la terapia individual se encuentra en un valor de 1,62 $(1,35-1,94)^{48}$, siendo la terapia grupal de $2,19(1,42-3,37)^{49}$ y la terapia aversiva de $1,98(1,36-2,90)^{50}$.

Los tratamientos farmacológicos con sustitutivos de nicotina han presentado una eficacia que oscila entre el 20\%-35\% en seguimientos de 6-12 meses de duración?. Por último, los programas comunitarios, de gran importancia desde el punto de vista de la salud Pública, parecen no estar en concordancia con los diseños de estudios de seguimiento de este tipo de intervenciones ${ }^{51}$.

\section{OBJETIVOS}

1. Presentar el diseño de un programa multicomponente, en el de tratamiento de dependencia a nicotina en el que se combina tratamiento farmacológico (Terapia Sustitutiva con nicotina combinada) y tratamiento psicológico con un enfoque sistémico -relacional complementario a las técnicas psicológicas clásicas del tratamiento psicológico cognitivo conductual.

2. Presentar los resultados de un estudio descriptivo de serie de casos ( $N=128$ ), después de un año de intervención clínica.

\section{METODOLOGÍA}

\section{Justificación de una visión sistémico - relacional de la demanda}

Desde nuestra experiencia clínica, los pacientes fumadores facilitan un shetting terapéutico que favorece la escucha activa y la organización de diseños de tratamiento individualizados a sus necesidades. Cuentan historias que se relacionan con su historia de vida $y$, en definitiva, con su historia de consumo de cigarrillos. En el caso de algunos fumadores, su pauta de consumo, así como su grado de dependencia, puede tener relación con una transmisión generacional asociada a reglas, mitos, roles, procesos de adaptación, procesos de individuación, de desvinculación, de vinculación.

Desde el enfoque del tratamiento de las adicciones con perspectiva sistémico-relacional, la abstinencia no es considerada un fin en sí misma. Ya Bateson, estableció la idea de contexto ${ }^{52}$, como el marco en el que las conductas se traducen en bits de comunicación que solo cobran significado en el contexto en el que se producen. Por lo tanto, la conducta (el síntoma), es 
causa y consecuencia de una serie de interacciones definidas en un sistema. De esta manera se produce un proceso de retroalimentación de la conducta, así como la co-evolución de la conducta en el proceso dinámico del propio sistema. Fumar se integra en la vida de la persona, etiquetándola, acompañándola, redefiniendo a través de la conducta de consumo las percepciones sobre resolución de crisis, de conflictos.

\section{Dos motivos que justifican la aportación sistémi- co-relacional}

La demanda asistencial dependerá en muchos casos del escenario terapéutico en el que se produce. Las personas fumadoras, hasta ahora, planteaban su problema como un conflicto ambivalente. "Tengo que dejar de fumar, aunque en realidad no tengo mucho interés por el cambio". La representación social del tabaquismo, los escasos recursos asistenciales, las relaciones tabáquicas entre asistentes y usuarios de los servicios, hacían que la demanda fuera poco intensa o escasamente motivada, al igual que el intento, poniendo especial hincapié en el esfuerzo personal y una ayuda farmacológica para quienes decidían que les era muy complicado dejar el consumo.

Cuando exploramos determinados grupos de fumadores nos encontramos con circunstancias muy distintas. Al menos hemos encontrado dos motivos suficientemente importantes como para generar un espacio reflexivo sobre el problema de la dependencia a nicotina.

\section{La recaída a medio-largo plazo.}

2. La necesidad de expresar conflictos o acontecimientos importantes en sus narrativas (historias contadas), que dificultan el proceso de cesación tabáquica hacia una abstinencia gratificante y normalizada.

\section{La recaída a medio - largo plazo}

Un estudio realizado sobre 72 pacientes pertenecientes al Servicio Cántabro de Salud, tuvo como finalidad conocer los motivos de recaída a largo plazo (más de un año de abstinencia) en los profesionales que demandaban ayuda para dejar de fumar o volver a dejar de fumar ${ }^{53}$, encontró que el 65,38\% ( $\left.n=51\right)$ había intentado dejar de fumar alguna vez. Estudiando este grupo, pudimos constatar que el 74,50\% $(n=38)$ habían recaído después de más de un año de abstinencia continuada, con una media de abstinencia de $15,24$ meses, mientras que el 25,49\% ( $n=13)$ habían recaído en un tiempo notablemente inferior al año, con una media de abstinencia continuada de 5,45 meses. El grupo de abstinentes de corta evolución presentaba unos motivos de recaída asociados principalmente a factores relacionados con el refuerzo positivo.
Del primer grupo, abstinentes de "larga duración", el $76,31 \%$ recayeron por causas de estrés negativo repartido de la siguiente manera: Crisis familiar (nuclear o de origen) resuelta por fractura (Separación, divorcio) (41,37\%); Crisis de ciclo vital (24,13\%); Pérdida de empleo (17,24\%); Duelo no finalizado (17,24\%)

\section{La verbalización de conflictos abiertos asociados a la percepción de dificultad para cesar con el consumo}

Otro estudio realizado sobre 113 pacientes ${ }^{54}$ tuvo como objeto conocer la existencia o no de demandas ocultas o de conflictos pendientes en las personas que acudían a un centro especializado en adicciones para dejar de fumar. La detección se realizó en 3 sesiones: primera entrevista, motivacional; entrevista evaluativa (en la que se incluyeron el genograma, la escucha activa, la connotación positiva y la narrativa del paciente como "sujeto-fumador") y la primera sesión de seguimiento. Los datos encontrados mostraron que el $63,71 \%(n=72)$ de los pacientes expresaron diferentes conflictos considerados por ellos como importantes o muy importantes para la continuidad de su comportamiento adictivo, más allá del deseo inicial de dejar de fumar. Clasificamos hasta 24 categorías de conflicto.

\section{TABLA 1 \\ Relación porcentual de problemas encontrados en la exploración diagnóstica.}

$-14,15 \%$ Crisis de pareja (conflicto no resuelto)

$-12,26 \%$ Soledad

$-10,37 \%$ Duelo no concluido

$-8,49 \%$ Dificultades de desvinculación de familia de origen en pacientes aparentemente emancipados (mandatos familiares)

$-6,60 \%$ Trastorno de ansiedad

$-6,60 \%$ Dificultades de desvinculación (convivencia con la familia de origen)

$-5,66 \%$ Divorcio en curso

$-4,71 \%$ Alcoholismo

$-3,77 \%$ Consumo de cannabis

$-3,77 \%$ Dificultades con hijos adolescentes

$-3,77 \%$ El pasado como presente (vivir de los recuerdos)

$-2,83 \%$ Timidez disfuncional

$-2,83 \%$ Acoso laboral

$-1,88 \%$ Malos tratos

$-1,88 \%$ Cuadro psicótico paranoide (colaboración con psiquiatría)

$-1,88 \%$ Depresión

$-8,46 \%$ Otros 
Como se puede observar en los resultados, los problemas más frecuentemente expresados son las dificultades de pareja en diferentes fases de ciclo vital, la soledad, en la que las pérdidas o la lejanía relacional han dado al cigarrillo un papel relevante como "figura acompañante". Los duelos no resueltos, por patológicos o por inconclusos como consecuencia de la proximidad de la pérdida también aparecen en los primeros puestos. A partir de aquí, pueden encontrarse verbalizaciones tan variadas como dramáticas, expresadas con claridad, emotividad y deseo de escucha. Acoso laboral, malos tratos, crisis de nido vacío, esquizofrenia, trastorno bipolar e incluso un caso de pérdida ambigua ${ }^{55}$-hijo en estado vegetativo- son experiencias vitales expresadas, como ya hemos dicho, en las 3 primeras sesiones de tratamiento.

Después de este breve análisis, consideramos que la apertura sistémico-relacional puede favorecer una mejor comprensión de la intensa relación tabaco-persona, permitiendo alternativas que generen un proceso más favorable a la normalización de la abstinencia.

\section{Características del programa terapéutico}

El programa diseñado para el abordaje clínico de la dependencia a nicotina, se desarrolla en un dispositivo especializado en drogodependencias. Tiene un año de duración desde la primera sesión, diagnóstica, hasta la sesión de finalización del programa. EI ALTA TERAPÉUTICA se realiza en una sesión en la que participan todas las personas que hayan contribuido directa o indirectamente a la consecución de los objetivos marcados al inicio del programa. Consta, en su temporalidad de 25 sesiones de psicoterapia, acompañada de tratamiento farmacológico, siempre con TSN combinada.

\section{Fases del programa de tratamiento}

1. Diagnóstico de dependencia a nicotina en todos los pacientes.

2. Prescripción de TSN combinada en todos los pacientes.

\section{Programa de tratamiento psicoterapéutico.}

\section{Diagnóstico de dependencia a nicotina}

No se es fumador desde hace dos días. No se fuma en momentos concretos en los que la sustancia tiene todo el peso de la recompensa o el poder reparador del sufrimiento. No se desarrolla un proceso de exclusión social por fumar. La dependencia a nicotina no se parece demasiado a los patrones de sufrimiento que se expresan en la demanda por consumo de otras drogas (cocaína, heroína, alcohol...). Todo es más sutil. El envoltorio de normalidad, de funcionalidad, brilla excesivamente en la demanda lineal. "Tengo que dejar de fumar...Y no puedo. No lo entiendo. Pero me veo con escasa fuerza de voluntad". Es por eso que hemos incluido técnicas que favorezcan la comprensión de la adicción entendida como un vínculo disfuncional entre cigarrillo y sujeto en su contexto de vida. El diagnóstico de dependencia a nicotina se ha realizado utilizando las siguientes técnicas y herramientas diagnósticas:

- Análisis de la actitud ante la demanda

- Entrevista motivacional

- Test de Fageström de Dependencia a Nicotina (TFDN)

\section{- DSM-IV-R}

- Valoración subjetiva de compulsión (craving)

- Desarrollo del genograma.

Las personas, cuando acuden pidiendo ayuda para dejar de fumar, pueden hacerlo con diferentes registros en cuanto a la expresión de urgencia o conflicto personal respecto a su conducta de consumo. Dejar de fumar no es una tarea sencilla. Puede parecerlo a la vista de algunos modelos de intervención que consideran que lo mismo que se ha adquirido una conducta, puede aprenderse otra. "Fumar vs. No fumar". Entre los fumadores se da el más notable nivel de incertidumbre respecto a sus expectativas abstinenciales. Aceptar su incertidumbre supone no limitarla, acompañarla. La confusión ${ }^{52}$ se convierte en un elemento real de deconstrucción, de desaprendizaje, para activar procesos de aprendizaje, de construcción de su propia realidad como no fumador.

El acercamiento al paciente fumador que vive su experiencia dependencial como problemática o inherente a su propia existencia sin saber realmente cuales son los beneficios de su íntima relación con la sustancia, debe realizarse pensando en restituir el poder al paciente y desmitificando el poder del cigarrillo en su propia vida. La intervención del terapeuta debe dejar suficiente espacio para generar autonomía en el proceso de descubrimiento de su realidad como no fumador. Whitaker lo expresa con una sencilla metáfora en la relación terapeuta- paciente, comparándola con la del entrenador y sus jugadores ${ }^{53}$, impulsando a éstos aumentar su resistencia física y usarse a sí mismos de una manera plena, con rendimientos que quizás ellos mismos no imaginan en sus constructos autolimitantes.

La Entrevista Motivacional la definimos, en este contexto terapéutico como un estilo de intervención que tendrá continuidad a lo largo de las diferentes sesiones, y que permite la apertura de la demanda hacia una perspectiva sistémico-relacional que permita la expresión de conflictos encubiertos. Este equipo clínico ha publicado las pautas de la Entrevista 
Motivacional específica para el trabajo preliminar con fumadores ${ }^{54}$

El interés del uso de estas herramientas diagnósticas radica en la complementación de un test específico de diagnóstico de dependencia a nicotina como lo es el TFDN que parece tener una potencia relativa, ajustada a determinados ítem, con los criterios diagnósticos de dependencia a sustancias DSM-IV-TR, que en el caso de nicotina cumple con extraordinaria coherencia para los diferentes ítem en la mayoría de los supuestos ${ }^{55}$.

Introducir en el contexto de intervención el craving como aproximación característica de compulsión, de pérdida de control, supone contextualizar dicho término como síntoma de base en las primeras fases del proceso. Las investigaciones sobre las expectativas frente al consumo, vinculadas a la acción de dopamina, pueden explicar la compulsividad en el consumo de cigarrillos. Parece que los incrementos de dopamina no están tan directamente relacionados con la recompensa, sino con la predicción de recompensa. Lo que Volkow y Li han dado en Ilamar relevancia ${ }^{56}$. Por lo tanto, la relevancia se referiría a la capacidad de ciertos estímulos o cambios ambientales para producir una activación o desencadenar un cambio atencional-conductual.

Respecto al uso del genograma, la familia puede ser considerada como el sistema primario y, salvo raras excepciones, más poderoso en el que una persona estructura su sentido de pertenencia. La mayoría de los conflictos verbalizados por los pacientes fumadores, se asocian directa o indirectamente con conflictos de índole familiar o bien con dificultades externas que afectan a los procesos de equilibrio y crisis del propio sistema, así como a la posición del paciente en dicho sistema. Aunque los genogramas han sido tradicionalmente utilizados desde el modelo sistémico relacional ${ }^{57,58}$, también se están empezando a manejar por clínicos de otras orientaciones ${ }^{59}$, incluso por parte de médicos de atención primaria que se sienten realmente "Médicos de Familia" y que, como consecuencia se acercan con interés al conocimiento sistémico.

El genograma es una representación gráfica del microcosmos familiar. Muestra su utilidad en la clínica del tabaquismo, al permitirnos profundizar sobre los factores ocultos inductores de la dependencia o facilitadores de la desvinculación tabáquica ${ }^{60}$. Así mismo, permiten descubrir la manera en la que los sucesos y las relaciones en las vidas de los pacientes están asociados con pautas de salud y enfermedad ${ }^{61}$.

El genograma está mostrando una notable utilidad en la redefinición de significados transgeneracionales de la dependencia, debido a que permite al paciente una observación en perspectiva de su posición a través de su historia familiar. El protagonismo relacional del tabaco puede aparecer con toda su potencia, tanto desde el pasado (familias fumadoras), como en el presente. Se desarrolla en la sesión evaluativa, quedando abierto para poder ser ampliado en sus datos a medida que avanza la terapia. En caso de conflictos relacionales puede también pedirse al paciente que elabore junto con el terapeuta un genograma alternativo idóneo, viable, en el que su posición sea más favorable.

\section{Prescripción de TSN combinada en todos los pacientes}

\section{Objetivos de la TSN}

a) Inhibición de los síntomas de abstinencia.

b) Generar un proceso de neuroadaptación inverso al generado en el proceso adictivo ${ }^{62}$.

Permitir que el cerebro pierda interés por nicotina a través de nicotina farmacológica, mediante dosis controladas y vías de administración lentas que limiten el poderoso reforzamiento que genera la vía de administración inhalatoria.

Se utilizaron parches de 24 horas (30/20/10, referido al tamaño del parche en $\mathrm{cm}^{2}$ ), en reducción progresiva cada 21 días. Las reducciones se realizaron de manera escalonada, mediante alternancia diaria las 72 horas anteriores a la reducción definitiva. 30-20-30-2020-.../20-10-20-10-10-.../10-0-10-0-0-.... El objetivo es disminuir el temor del paciente ante las reducciones, generalmente más acentuado en la primera reducción.

La oportunidad de utilización de terapia nicotínica oral (chicles de nicotina de 2-4 mg o comprimidos de nicotina de $1 \mathrm{mg}$ ) tiene como objetivo la corrección subjetiva de craving, como síntoma por excelencia del proceso de abstinencia y que produce la mayoría de los consumos puntuales inductores del aumento de probabilidad de recaída. Durante la experiencia craving el estado emocional es disfórico, describiendo los pacientes sus estados como tensos, inquietos, ansiosos o frustrados ${ }^{63}$. Un modelo descriptivo que recoja el craving como el síntoma "negativo" por excelencia, supone la aceptación del afrontamiento de momentos puntuales de desasosiego que acompañan a las dificultades propias de la aceptación de la cesación tabáquica.

\section{Programa de tratamiento psicoterapéutico}

Las aportaciones del Modelo Sistémico- Relacional al tratamiento del tabaquismo, permiten explorar la organización del sistema familiar (nuclear y de origen) del paciente identificado, nos permite establecer un posible diagnóstico de disfuncionalidad latente en el seno de la estructura familiar ${ }^{64}$ (crisis abiertas no 
resueltas, límites difusos o rígidos, alianzas o coaliciones), siempre que dichas dificultades se expresen como problemas potenciales para la cesación tabáquica. No se trata, desde nuestra modalidad de intervención, de realizar Terapia de Familia en cada caso, sino de tener una visión relacional del problema. El fumador integrado como subsistema en un "sistema - fumador", y éste a su vez, en un "contexto-fumador".

El paciente fumador es, posiblemente, la persona con un problema adictivo que más incomprendida se siente. Por eso, consideramos de utilidad en el encuadre terapéutico el desarrollo del joining, transmitiendo al paciente la comprensión, el respeto, la escucha interesada, nuestra curiosidad. Hacer sentir al paciente que todo lo que nos diga es importante y que estamos deseando escucharle. Para ello sistematizamos en la intervención las siguientes técnicas:

\section{- Preguntas abiertas}

Las primeras verbalizaciones de los fumadores suelen ser lineales, porque en la mayoría de los casos proyectan, "visualizan" lo que va a ocurrir. Creen saber cómo se les va a ayudar, pues están informados de los diferentes "métodos para dejar de fumar". Su relación adictiva con la sustancia tiene bases emotivas y cognitivas poderosas. "Me gusta fumar", "Fumar es un placer", "Fumar me tranquiliza", "Fumar me acompaña", “No entiendo por qué no puedo quitarme este hábito", etc.

Mostrar nuestro interés por las construcciones cognitivas que los fumadores realizan de su relación con el tabaco permite reflexiones relacionales alternativas:

- "Tengo la extraña sensación de que usted debe sentirse muy solo". (Fumadores compulsivos con afectación orgánica y percepción autodestructiva, ambivalentes ante la idea de dejar de fumar).

- "Estoy muy interesado en los beneficios que obtiene del tabaco".

- "Me gustaría saber qué cree que piensa su familia (pareja, hijos, sistema familiar en su conjunto) de su decisión de dejar de fumar".

\section{- Modificación de los constructos cognitivos}

En el caso de la dependencia a nicotina, la apertura terapéutica hacia redefiniciones de su forma de entender la conducta tabáquica, se dan en las primeras sesiones, siempre que hayamos conseguido entender el contexto social y familiar del paciente fumador ${ }^{65}$.

- Es muy posible que su padre le legara algo más que el consumo de cigarrillos. Al igual que su abuelo le transmitió algo más a su padre que el fumar".
- "Parece que acepta la suerte de su padre, y de su abuelo...¿ ¿ Qué piensa de esto su mujer?"

- "Creo que está usted en condiciones de liberar a los fantasmas de su familia del mito: Todos los varones mueren fumando... ¿O por fumar?"

También, en el caso del tabaco, el consejo profesional, o las explicaciones divergentes son interesantes, como consecuencia de la riqueza de matices que se mantienen escondidos en las representaciones sociales y cognitivas de los fumadores y de los miembros de su familia.

* La presencia de la familia o parte de la familia en sesión. La posibilidad de participación.

La demanda asistencial no siempre es realizada por el paciente identificado. En muchos casos es la pareja la que pide ayuda, y se presenta en la primera sesión. Se invita a la participación en la sesión de los miembros de la familia que acuden, con la finalidad de identificar las posiciones de cada miembro respecto al problema motivo de la demanda.

En este sentido, la presencia de familiares del fumador, suele estar marcada por la etiqueta de "no fumador" del otro o de los otros, en muchos casos hijos/hijas preadolescentes, aliadas con el cónyuge no fumador para ejercer presión sobre la posibilidad de modificación de la conducta.

La demanda resulta en muchos casos más urgente para el resto de los familiares que para el propio fumador. En esta situación se hace imprescindible evitar las resistencias y conflictos ya representadas con mucha probabilidad en el escenario familiar. La connotación positiva, centrada en la redefinición del problema alivia y serena el ambiente comunicacional.

-"Resulta emocionante comprobar cuanto le quiere su familia, el empeño que ponen en evitar que desaparezca de sus vidas".

- "Parece que es usted imprescindible en su familia, a tenor de esta intensa preocupación por su vida".

La redefinición de las dificultades abstinenciales del paciente pasa por aceptar el papel que juega el tabaco en la interpretación funcional del sujeto, para después comenzar el proceso de acercamiento terapéutico que permita una exploración conjunta (terapeuta- paciente) de las dificultades, teniendo como brújula el mapa familiar66.

* Intervención estratégica sobre el síntoma fumar

Siguiendo la evolución de los estudios de M. Erickson y Jay Haley 67, 68, 69, 70, así como su forma de entender la terapia contextualizada a través de la teoría de los sistemas y la teoría de la comunicación ${ }^{71,72}$, pode- 
mos comprender mejor algunos aspectos entendidos como disfuncionales por parte de algunos fumadores que tienen dificultades para conseguir una abstinencia gratificante y normalizada. Si "fumar", lo consideramos una forma de etiquetaje para una serie de comportamientos, dentro de una o varias organizaciones relacionales, el sujeto fumador integra de forma normalizada su vínculo adictivo en estos escenarios. Desde una perspectiva estratégica, el problema podríamos redefinirlo. No es "fumar", sino "intentar dejar de fumar", una y otra vez. Las dificultades abstinenciales de carácter psicológico, el incumplimiento de las expectativas respecto al cambio puede llevar a una recaída elaborada incluso previamente al consumo, lejana del sentimiento culpabilizador que producen otras adicciones, renueva el equilibrio relacional, pero aumenta las resistencias para el cambio en algunos fumadores. Desde un enfoque estratégico, se ordenan y priorizan las dificultades buscando soluciones una a una. El uso parcial de este tipo de intervención, permite la vista terapéutica hacia el futuro, vinculando al paciente en un "viaje exploratorio abstinencial", que supone el reencuentro emocional y relacional desde su óptica de no fumador.

\section{a) Utilización de elementos de la terapia breve.} Terapia centrada en las soluciones

Existen tantas dificultades en el abordaje del tabaquismo, como fumadores que solicitan ayuda. Al contrario que en otras adicciones, el fumador se ve en muchas ocasiones solo ante su conflicto. Incomprendido y sin poder comprender qué es lo que impide su cesación, ha intentado muchas veces dejar de fumar. Intentos que le han hecho creer que nunca podrá dejar una conducta que puede costarle la vida. Es por eso que cuando señala la dificultad en la conducta tabáquica, puede ser de mucha utilidad reflexionar con el paciente desde la perspectiva de intervención centrada en las soluciones.

Su gestor intelectual, Steve de Shazer, evoluciona progresivamente hacia la importancia del lenguaje, definiendo el sistema como "un sistema lingüístico", siendo las palabras el elemento más importancia en la transacción terapéutica ${ }^{73,74}$

Los elementos que nos ayudan a enfatizar sobre las soluciones en un paciente con dependencia a nicotina desde este enfoque, son:

1) Prestar la atención sobre las capacidades de los pacientes para gestionar sus problemas. Los fumadores (incluso los más compulsivos), muestran una amplia gama de comportamientos saludables, vinculados con una adecuada higiene mental. Así, es interesante trabajar sobre la idea de "salud mental", disminuyendo el impacto de la patología, de la enfermedad mental ${ }^{75}$.
2) La utilización, como elemento constitutivo de la terapia para la solución, supone extraer del paciente sus capacidades, recursos y atributos saludables para resolver el conflicto en proceso. Los fumadores, en muchos casos recurren a mediaciones casi milagrosas, convencidos de la imposibilidad de conseguir un objetivo tan complejo como dejar de fumar. Su percepción de escasa autoeficacia redunda negativamente en la expresión de sus propias capacidades para producir la desvinculación.

3) Debemos atender a la percepción que tiene el propio paciente del problema. Por lo tanto, la dependencia a nicotina, se representa de forma tan variada como posibles pacientes fumadores que piden ayuda.

4) Parquedad. De Shazer acostumbraba a citar las palabras de Guillermo de Ockham: "Lo que se puede hacer con pocos medios falla si se hace con muchos"76.

5) El cambio es inevitable. Las personas se encuentran en constante cambio. Incluso, respecto a su consumo de cigarrillos las personas modifican sus pautas. Antes de llegar a consulta, muchos de ellos han realizado cambios, intentos de abstinencia, poniendo a prueba su relación con la droga. Se trata de trabajar sobre las excepciones, planteando el hecho de que el problema no ocurre de manera constante. Hay momentos en la vida del paciente en los que fumar no es lo prioritario, no es lo fundamental. Incluso, pareciéndolo, otras prioridades hacen que fumar quede minimizado, pudiendo atribuir a estos escenarios el rol de "no fumador"77.

Dejar de fumar, como hemos dicho anteriormente, debe ser un viaje exploratorio.

Para ello, puede ser útil en determinados pacientes, la pregunta que se ha denominado "del milagro"73, que permite vislumbrar una realidad futura sin tabaco.

Otro tipo de preguntas que resultan útiles son las preguntas de escala ${ }^{77}$, aquellas que permiten el análisis reflexivo de estereotipos relacionados con el consumo de cigarrillos. La deconstrucción de la percepción de placer, la gestión de estrés y ansiedad, la propia percepción negativa de ansiedad en el manejo de contingencias interpersonales son aspectos en los que el manejo de las preguntas de escala puede motivar la relativización de los mitos integrados por parte del paciente en su relación con el cigarrillo.

\section{b) El diálogo estratégico}

Los problemas del hombre son considerados como el producto de la interacción entre el individuo y la rea- 
lidad, por lo que, desde esta perspectiva, el enfoque no tiene como base "por qué existe el problema", sino sobre "cómo funciona el problema" y especialmente sobre "qué hacer" para resolverlo, guiando a la persona a cambiar no solo sus propios comportamientos sino también sus propias modalidades perceptivas. El diálogo es entendido, desde este enfoque, como "un encuentro de inteligencias", o "inteligencia a dos"78. El diálogo estratégico se configura en el tratamiento del tabaquismo, con la siguiente secuencia:

1. La solución intentada. Este elemento es el eje de las construcciones terapéuticas desarrolladas por el grupo del Mental Research Institute (MRI) de Palo Alto $^{79,80}$. Las soluciones intentadas son las reacciones y comportamientos llevados a cabo por las personas para afrontar las dificultades en la relación consigo mismas, con los demás y con el mundo; reacciones y comportamientos que en muchas ocasiones complican más que resuelven y que terminan por volverse rígidos, en modelos redundantes disfuncionales de interacción con la propia realidad del paciente. El comportamiento disfuncional es la reacción que la persona cree mejor para una situación determinada, lo cual nos lleva a una interesante reflexión: "el problema existe precisamente en virtud de lo que se ha hecho para intentar resolverlo". Podemos hablar de la retroalimentación del problema a través de las soluciones fallidas, algo que podemos constatar en los intentos de las personas que presentan y sienten un problema con su conducta tabáquica. Muchas veces han intentado dejar de fumar, pero las pautas han sido más o menos las mismas, aunque las formas hayan sido diferentes.

Las preguntas no tienen como objetivo guiar al clínico hacia la comprensión del problema a resolver. Son, desde este modelo, el vehículo para inducir al paciente a "sentir" las cosas de modo diferente $y$, en consecuencia, a cambiar sus reacciones, descubriendo sus recursos que estaban bloqueados por las percepciones anteriores, rígidas y patógenas.

2. Las preguntas con ilusión de alternativa. En el diálogo estratégico, la pregunta se estructura con dos posibilidades opuestas de respuesta y el interlocutor podrá "decidir" cual de las dos se adapta a su caso. Por lo tanto, en el arte de la persuasión (que sería imprescindible en el caso de una buena investigación, de una búsqueda diagnóstica, o bien de respuestas favorecedoras de cambios), la secuencia de preguntas actúa como un embudo, haciendo que la persona confluya, a través de sus respuestas, al punto clave de sus aserciones precedentes. Todo de tal forma que el paciente llegue a sentir la exigencia de cambiarlas, gracias a las nuevas percepciones descubiertas dentro del diálogo, y sustituyéndolas por las nuevas.

Las preguntas con ilusión de alternativas parten de interrogaciones, primero más generales, que después, en un proceso de espiral, se estrechan, sobre la base de las respuestas, estructurándose en torno a las particularidades de las situaciones y poniendo en evidencia los puntos críticos potenciales.

3. Las paráfrasis reestructurantes. La paráfrasis reestructurante se define como una maniobra que sigue a la secuencia de dos o tres preguntas: se utilizan las respuestas del paciente para formular una definición del problema que verifique la correcta comprensión. En ella no se propone valoración ni interpretación alguna. El proceso busca un equilibrio en el diálogo, desde la humildad del clínico, dando pie al paciente a que sea el verificador de sus problemas en la boca del terapeuta:

\section{"Corríjame si me equivoco: ateniéndonos a todo lo que usted ha afirmado, parece que...."}

El paciente se convierte en el guía de la conversación y se le considera como el verdadero experto del problema, pues es SU problema y la vivencia de su problema lo que activa búsqueda de soluciones. Parafraseando las dos o tres respuestas a las anteriores preguntas estratégicas, se da a la persona la sensación de ser respetado y no obligado, y considerado más que descalificado.

4. Evocar sensaciones. Dialogar estratégicamente significa inducir cambios en el interlocutor mediante aquello que se le hace sentir; por lo tanto, el recurso al lenguaje evocativo se convierte en esencial. Pudiendo utilizar todas las figuras retóricas y poéticas posibles, lo importante es que la formulación comunicativa provoque el efecto evocador planificado en el interlocutor.

Que se utilice un aforismo o una metáfora (en el tratamiento del tabaquismo hemos elaborado algunas metáforas de uso sistemático en la entrevista motivacional ${ }^{54}$ ), una anécdota o un ejemplo concreto, una cita poética o una narración, una argumentación o un contrasentido, es igual, con la condición de evocar la sensación que active el efecto emocional idóneo para el fin persuasivo. El éxito de la utilización de esta modalidad reside en orientar sus efectos en dirección opuesta respecto a actitudes y comportamiento que han de interrumpirse o cambiarse, y de forma amplificadora positiva respecto a aquellas reacciones que se quieren incentivar o incrementar. Por lo tanto, la dificultad de esta técnica radica en ser utilizada mediante un estilo comunicativo adecuado a las características personales del paciente.

5. Resumir para redefinir. Este resumen, que será una secuencia articulada de las respuestas de la persona, tiende a redefinir de forma concluyente los descubrimientos que hemos hecho de forma conjunta respecto al problema que el paciente ha presentado, a su persistencia y, sobre todo, a las soluciones. Mediante el proceso de redefinición final se crea una sensación tranquilizante de conocimiento respecto al 
problema y a su percepción, además de aquello que se hace necesario para su solución.

6. Prescribir como descubrimiento conjunto. La fase de las indicaciones prescriptivas representa un momento fundamental, siendo, además colofón de la sesión. Deben estar, las prescripciones, en consonancia con lo descubierto y acordado a través del diálogo terapéutico. Las tareas deben ser invitaciones formales, caracterizadas por el clínico como de importancia para establecer la solidez del compromiso dialéctico que se ha generado a través de la sesión. Pueden ser muchas y de muy diferentes tipos. Desde autorregistros relacionados con las horas en las que aparece craving, hasta cartas de despedida o bienvenida al tabaco o a la abstinencia, cuadernos de control de experiencias placenteras sin fumar, estudio del placer a través de preguntas de escala, etc.

Se debe hacer constar lo complicado de la tarea, reconociendo dudas sobre la posibilidad de realización por parte del paciente, que, en general despiertan aún más el deseo de cumplimiento del paciente.

\section{* Una aproximación a la narrativa de los fumadores}

Un fumador expresaba su relación dialéctica con el cigarrillo como el elemento mediante el cual era capaz de describir el mundo, la realidad, sus sueños, sus fantasías. El cigarrillo parece definir parcialmente su identidad a través de su relación con el tabaco, de la integración de la sustancia en sus construcciones de la realidad, en sus interacciones con el contexto exterior. "Soy fumador desde siempre." "Fumo toda la vida". "El tabaco es mi felicidad" "Mi arte depende del tabaco" ... Toda expresión es expresión de un observador ${ }^{81,82}$. La realidad terapéutica es expresada progresivamente por el fumador, solo cuando entiende que es entendido, cuando al marcar una dirección en el mapa de la realidad, contempla la detallada observación del observador como acompañante en las rutas que afloran al describir la intensa presencia del tabaco en su relación con el mundo.

La presencia cada vez más persuasiva de las tecnologías de "saturación social"83, parece indicar que las personas tienden a depender de ellas mismas. Definen sus realidades y sus necesidades, sus relaciones y premisas vitales, entre las que puede asentarse fumar.

Gergen $^{84}$ parece describir la solución a la crisis de la posmodernidad a partir del construccionismo social. Un paradigma intelectual que nos acerca a las narrativas personales como cemento de nuestra propia identidad. El construccionismo otorga relevancia a la conversación en la construcción de la identidad, y el lenguaje y los significados aparecen con potencia como activadores de proceso. Algunos fumadores, en este sentido, presentan dificultades a la hora de expresar, hablar de sí mismos como personas no fumadoras en el futuro. En poco más de 3 años, la representación social, así como el discurso social relacionado con el tabaco, ha dejado sin contexto a una gran cantidad de fumadores. Pero se consolidan en su relación con el tabaco, lo que se denominaría sus "narrativas como fumadores", expresando una reafirmación en su conducta de consumo al sentir un ataque a su identidad, a sus necesidades y costumbres, hasta hace poco tiempo aceptadas.

\section{La terapia como narración}

Solo algunos apuntes de nuestras impresiones sobre la utilidad del modelo narrativo de Michael

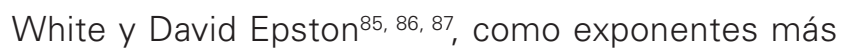
destacados de esta forma de entender la interacción terapéutica. Muchos fumadores han realizado numerosos intentos para dejar de fumar, comprobando con angustia la dificultad para aceptar su nueva situación.

“La persona no es el problema. El problema es el problema". Podemos destacar desde nuestra reflexión terapéutica con fumadores que desean dejar de fumar, tres aspectos relevantes del modelo narrativo:

\section{La externalización del problema}

Empleada en el trabajo con pacientes fumadores intensivos, aquellos que integran la dependencia en todos los planos de su vida. Esta herramienta supone el distanciamiento del tabaco. Permite pues, la diferenciación del paciente en situación de no consumo, de aquel momento en el que el tabaco se hace presente de manera intensa en la vida del fumador. Podemos acercarnos al paciente en su viaje abstinencial con una deconstrucción que produzca una alternativa: Compulsión (adicción) por reflexión previa (no- adicción).

\section{La objetivación del problema}

El paciente tiene la oportunidad de comprender el problema a través de las condiciones culturales e históricas que han dado origen a la integración del consumo como una conducta habitual. La agresión del consumo de cigarrillos puede llegar a resultar evidente para el fumador, pero solo si está en juego un proyecto, una alternativa, el cumplimiento de unas expectativas alternativas a su condición de fumador, el paciente favorecerá el cambio.

\section{Narrativas dominantes vs. narrativas alternativas}

Partir de la base de un mundo constituido a través de una red de relatos o narrativas múltiples, implica, aceptar la posibilidad de que dichas narrativas, en diferentes contextos de vida, entretejen la consciencia de nosotros mismos. ${ }^{88,89}$

Desde esta perspectiva, el lenguaje no es representacional. La realidad consiste y se expresa en las descripciones que cada uno hace de los hechos, de las vivencias, de las personas, emociones y experiencias. 
El paciente fumador construye la realidad desde su autoimagen como "persona fumadora". Habla la voz del fumador, a través del borrador vital utilizado desde la adolescencia. No entrando en más reflexiones sobre las deficiencias habidas hasta hace pocos años del control del tabaquismo, la narrativa permite la invitación a la creación de una redefinición de la abstinencia, que se convierte en el principio, no en el final. Permitir la posibilidad de que hable "la voz del no fumador", supone la deconstrucción de mitos, de gestión de la vida con la "imprescindible e inevitable presencia del cigarrillo".

Con todo, en el programa se aplican los criterios del tratamiento psicológico cognitivo conductual, integrando aspectos de diferentes modelos en beneficio de una más completa y exitosa intervención. La acción terapéutica en recaída, el trabajo sobre la fantasía del consumo controlado, o la identificación de situaciones de riesgo, son consideradas actuaciones de base del modelo de tratamiento presentado.

\section{MUESTRA}

Estudio descriptivo de 128 pacientes fumadores que solicitan ayuda para dejar de fumar en centro especializado de adicciones (Instituto Cántabro de Conductas Adictivas), en la población de Santander (Cantabria).

Edad. La edad media de los pacientes es de 41.78 años, con una desviación típica de 8.92 años. El paciente más viejo tiene 82 años y el más joven 20. La distribución de edades es muy semejante en hombres y en mujeres, tal y como se muestra en la siguiente gráfica.

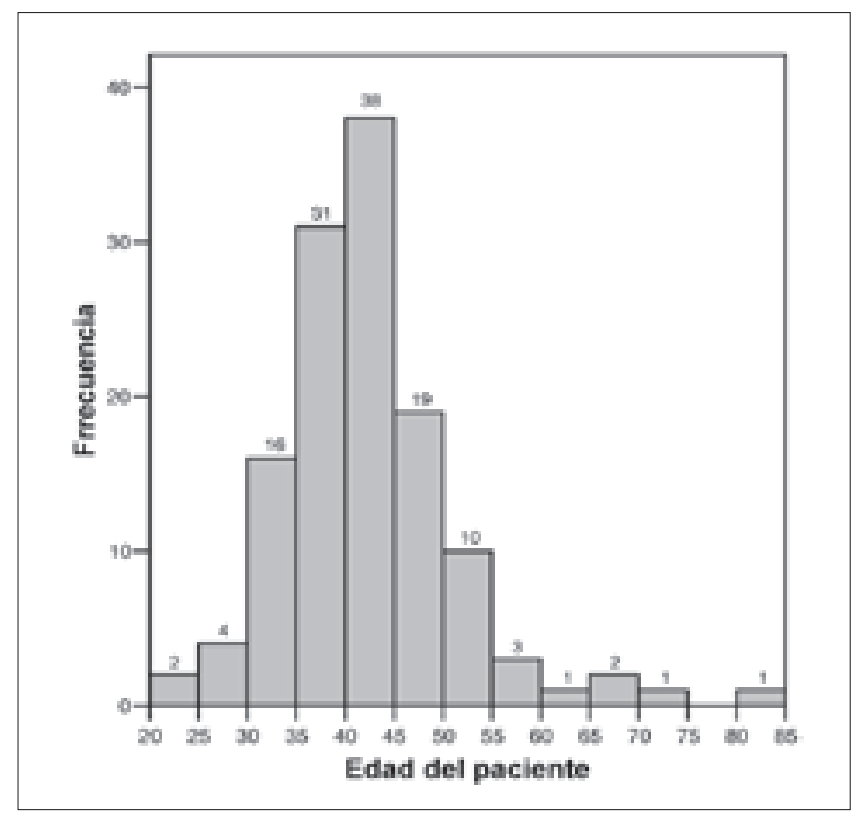

FIGURA 2

Histograma de la distribución de la edad

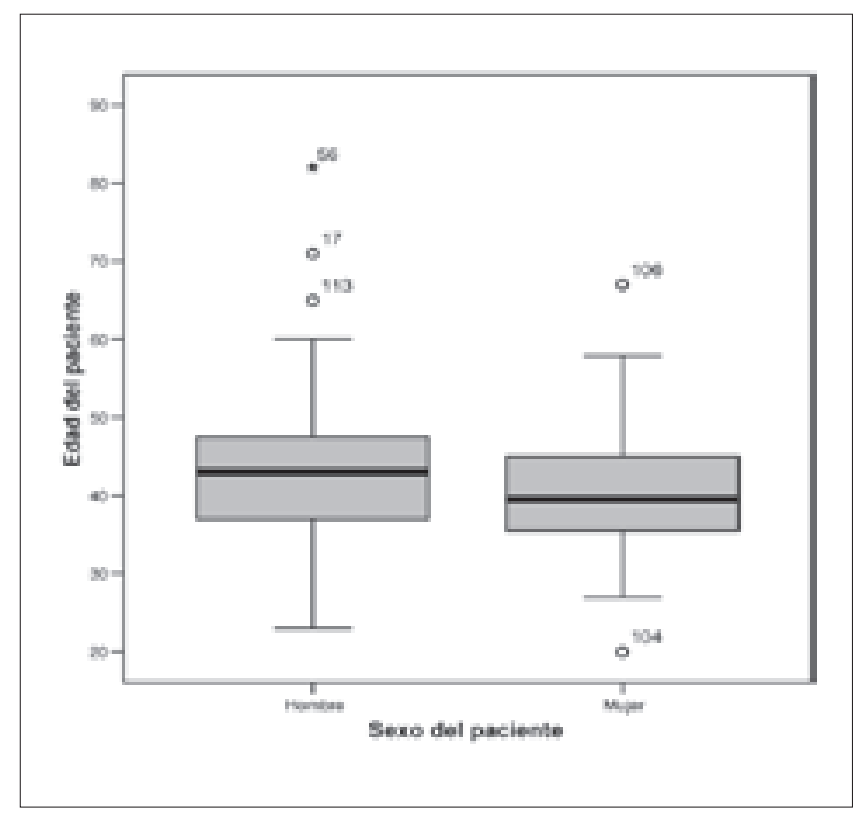

FIGURA 3

Distribución de edad por sexos

Número de cigarrillos fumados al día. El consumo medio de cigarrillos es de 30,04 cigarrillos día. El $50 \%$ de los pacientes fuman entre 20 y 20 cigarrillos al día. El consumo de cigarrillos es ligeramente inferior en mujeres que en hombres. El número de cigarrillos medio en hombres es de 33.52 mientras que en mujeres es de 26.56. La dispersión del número de cigarrillos es mayor en hombres con una desviación típica de 11.91 y un rango intercuartílico de 15 cigarrillos, frente a 7.46 y 10 en mujeres, respectivamente.

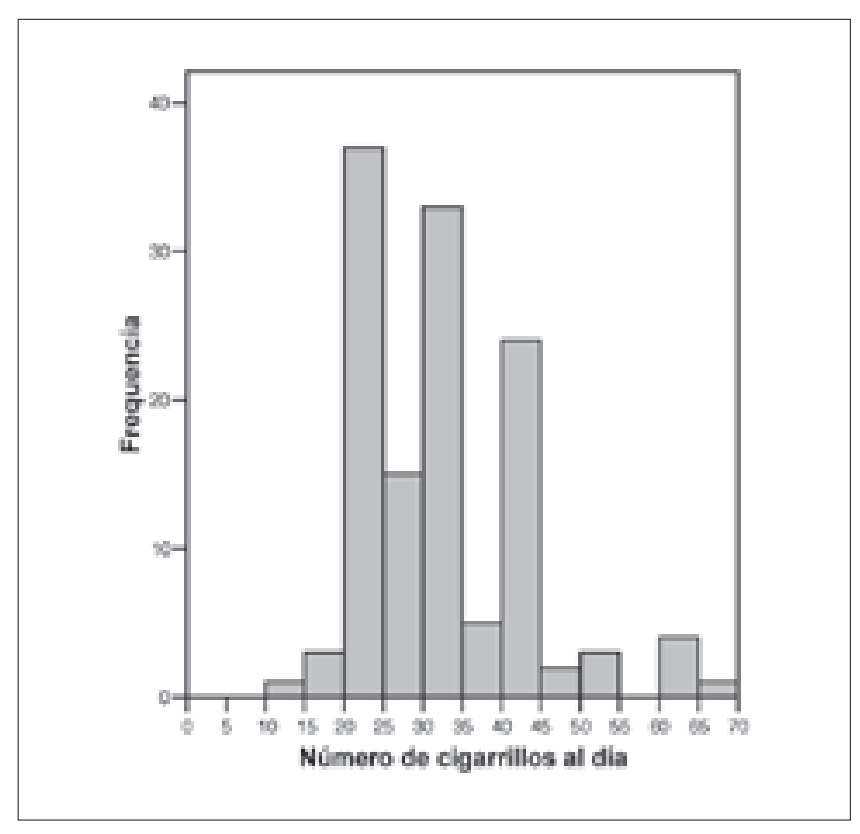

FIGURA 4

Histograma de la distribución del consumo de cigarrillos diarios entre los pacientes estudiados 
Años de consumo activo. La muestra estudiada presenta una larga evolución de consumo de cigarrillos, sin presentar diferencias significativas por sexos. 27,03 años de consumo en los hombres frente a los 23,05 años en las mujeres.

Intentos de cesación tabáquica. En cuanto a los intentos para dejar de fumar, En la tabla siguiente se muestra la distribución del número de intentos de dejar de fumar, que también se representa en el histograma de la Figura 5.

Después de codificar el numero de intentos en tres categorías, 0 intentos, 1 intento y 2 ó más intentos, la siguiente tabla muestra que el 34,4\% de los pacientes no ha intentado nunca dejar de fumar, el 28,9\% lo ha intentado una vez y el resto $(36,7 \%)$ lo ha intentado dos o más veces.

Test de Fagerström de Dependencia a Nicotina Modificado. EITFDNM se ha recogido esta variable en 127 casos. La frecuencia de las puntuaciones TFDN se presentan en la tabla siguiente (Tabla 3).

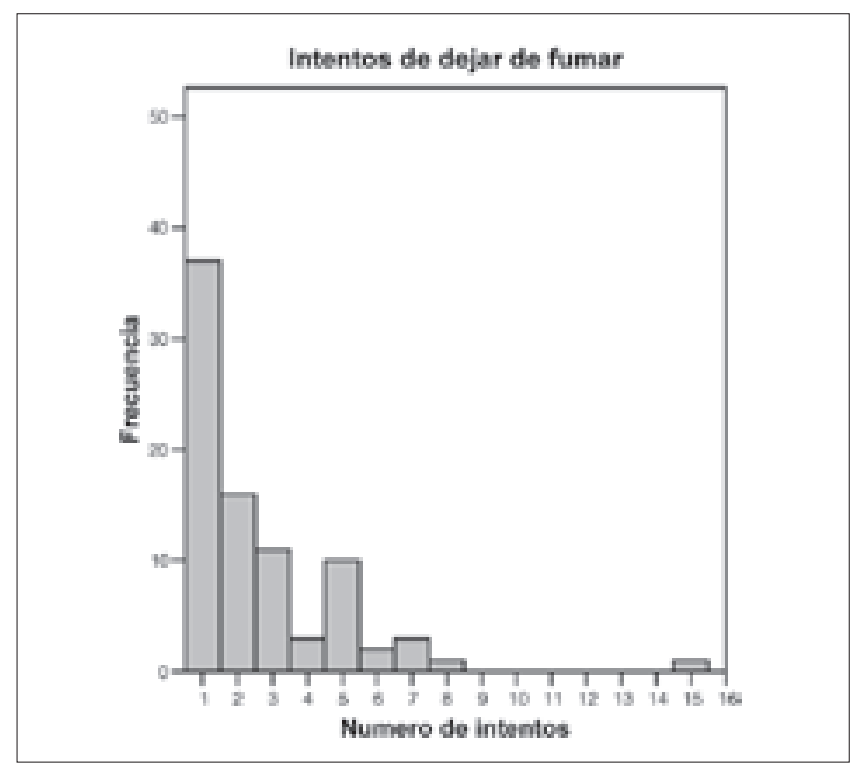

FIGURA 5

Intentos de cesación del grupo de pacientes estudiados.

TABLA 2. Número de intentos de dejar de fumar por sexo.

\begin{tabular}{|c|c|c|c|c|c|c|}
\hline \multirow{3}{*}{$\begin{array}{l}\text { Intentos de } \\
\text { dejar de } \\
\text { fumar }\end{array}$} & \multirow{2}{*}{\multicolumn{2}{|c|}{ TOTAL }} & \multicolumn{4}{|c|}{ Sexo del paciente } \\
\hline & & & \multicolumn{2}{|c|}{ Hombre } & \multicolumn{2}{|c|}{ Mujer } \\
\hline & Casos & $\%$ & Casos & $\%$ & Casos & $\%$ \\
\hline TOTAL & 128 & $100,0 \%$ & 64 & $100,0 \%$ & 64 & $100,0 \%$ \\
\hline 0 & 44 & $34,4 \%$ & 20 & $31,3 \%$ & 24 & $37,5 \%$ \\
\hline 1 & 37 & $28,9 \%$ & 19 & $29,7 \%$ & 18 & $28,1 \%$ \\
\hline 2 & 16 & $12,5 \%$ & 6 & $9,4 \%$ & 10 & $15,6 \%$ \\
\hline 3 & 11 & $8,6 \%$ & 6 & $9,4 \%$ & 5 & $7,8 \%$ \\
\hline 4 & 3 & $2,3 \%$ & 2 & $3,1 \%$ & 1 & $1,6 \%$ \\
\hline 5 & 10 & $7,8 \%$ & 6 & $9,4 \%$ & 4 & $6,3 \%$ \\
\hline 6 & 2 & $1,6 \%$ & 1 & $1,6 \%$ & 1 & $1,6 \%$ \\
\hline 7 & 3 & $2,3 \%$ & 2 & $3,1 \%$ & 1 & $1,6 \%$ \\
\hline 8 & 1 & $8 \%$ & 1 & $1,6 \%$ & 0 & $0 \%$ \\
\hline 15 & 1 &, $8 \%$ & 1 & $1,6 \%$ & 0 & $0 \%$ \\
\hline
\end{tabular}

TABLA 3. Puntuación del Test de Fagerström de Dependencia de Nicotina (TFDN), por sexo.

\begin{tabular}{|c|c|c|c|c|c|c|}
\hline & \multirow{2}{*}{\multicolumn{2}{|c|}{ TOTAL }} & \multicolumn{4}{|c|}{ Sexo del paciente } \\
\hline & & & \multicolumn{2}{|c|}{ Hombre } & \multicolumn{2}{|c|}{ Mujer } \\
\hline & Casos & $\%$ & Casos & $\%$ & Casos & $\%$ \\
\hline TOTAL & 127 & $100,0 \%$ & 63 & $100,0 \%$ & 64 & $100,0 \%$ \\
\hline 1 & 1 & ,8\% & 0 & $0 \%$ & 1 & $1,6 \%$ \\
\hline 2 & 1 &, $8 \%$ & 0 & $0 \%$ & 1 & $1,6 \%$ \\
\hline 3 & 8 & $6,3 \%$ & 4 & $6,3 \%$ & 4 & $6,3 \%$ \\
\hline 4 & 13 & $10,2 \%$ & 7 & $11,1 \%$ & 6 & $9,4 \%$ \\
\hline 5 & 22 & $17,3 \%$ & 10 & $15,9 \%$ & 12 & $18,8 \%$ \\
\hline 6 & 33 & $26,0 \%$ & 16 & $25,4 \%$ & 17 & $26,6 \%$ \\
\hline 7 & 19 & $15,0 \%$ & 8 & $12,7 \%$ & 11 & $17,2 \%$ \\
\hline 8 & 23 & $18,1 \%$ & 16 & $25,4 \%$ & 7 & $10,9 \%$ \\
\hline 9 & 3 & $2,4 \%$ & 0 & $0 \%$ & 3 & $4,7 \%$ \\
\hline 10 & 4 & $3,1 \%$ & 2 & $3,2 \%$ & 2 & $3,1 \%$ \\
\hline
\end{tabular}


Como era de esperar, el gráfico de barras siguiente muestra que las frecuencias de la escala TFDN se ajustan a una campana de Gauss escorada a la derecha, típico en una muestra de consumidores habituales de tabaco.

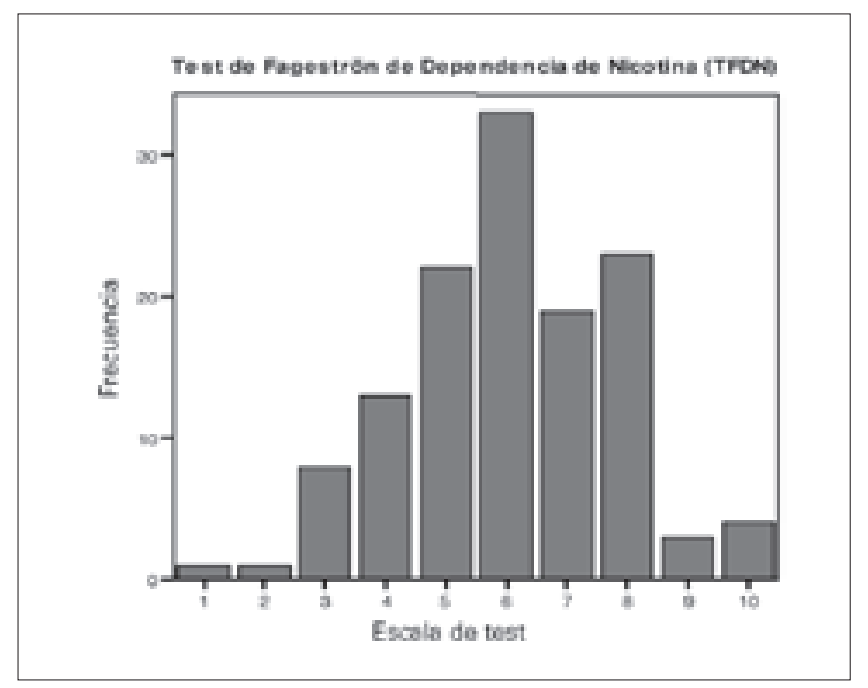

FIGURA 6

Valor delTFDN en el grupo de pacientes estudiados.

Sin embargo, al exponer a los pacientes a los criterios de dependencia según DSM-IV-TR, todos, el $100 \%$ cumplen con criterios de dependencia a nicotina (F17.2X) [305.1]. El 76,36\% de los pacientes evaluados cumplen con 4-5 criterios de dependencia.

Variables sociales. El número de pacientes que tienen pareja es 103, de los cuales el 55,3\% tienen pareja fumadora. Este porcentaje sube al 59,6\% entre las mujeres. El 40,6 \% de los pacientes no tienen hijos, el 13,3\% tienen uno solo y el $46,1 \%$ el tienen 2 o más. Las diferencias por sexo no son significativas. Solo el 13,2\% tienen algún hijo que sea fumador. Este porcentaje baja al 7,9\% entre los hombres y sube al $18,7 \%$ entre las mujeres.

Presencia de patología asociada al consumo de tabaco. El 82,5\% de los pacientes no presentan ninguna patología orgánica asociada al tabaquismo.

Presencia de psicopatología. De manera análoga, la mayoría de los pacientes (85.2\%) no presentan ninguna psicopatología asociada al tabaquismo. (COMORBILIDAD PSIQUIATRICA).

Valores de monóxido de carbono en aire espirado (CO ppm). La serie de pacientes estudiados tiene una concentración de $\mathrm{CO}$ de 21,90 ppm prueba tomada en aire espirado mediante cooxímetro Mini 2 Smokerlyzer, con un rango de medición de 0- 500 ppm y lectura simultánea de CO ppm y \% COHb (carboxihemoglobina). La toma de muestra se realizó el día de evaluación diagnóstica, así como el día de alta terapéutica.
Se ha realizado el análisis de otras muchas variables, como el motivo principal de la demanda, los Problemas Relacionados Con Medicamentos (PRM) de la TSN, la evolución del sueño durante el proceso terapéutico, la tendencia de la percepción subjetiva del craving o la presencia o no de consumos esporádicos en el tiempo de tratamiento. Sin embargo consideramos que su exposición excede los objetivos de este trabajo.

Criterios de Exclusión. Solo se aplicaron dos motivos de exclusión:

-Embarazo.

-Otras patologías adictivas en remisión parcial según criterios DSM-IV-TR, considerando la posibilidad de desestabilizar el proceso abstinencial a la droga anteriormente tratada.

Los casos de psicopatología diagnosticada en el momento de petición de demanda asistencial en nuestro centro, se abordaron en red, con la colaboración de los servicios de salud mental de referencia del paciente.

Coste del tratamiento. El tratamiento ha tenido un coste para el paciente de $500 €$. En este precio estuvo incluida la TSN combinada, así como la intervención psicoterapéutica desarrollada.

\section{RESULTADOS}

\section{Criterios de alta terapéutica}

La consideración de ALTA TERAPÉUTICA se relaciona con los siguientes criterios:

a) Ausencia de terapia farmacológica nicotínica. En determinados casos, no incluidos en este trabajo, puede valorarse un programa de reducción de daños dependiendo de las dificultades del paciente y de los objetivos consensuados con el equipo de intervención.

b) Ausencia de compulsión, de proyección de pensamientos futuros de consumo o de consumos puntuales, al menos en los seis últimos meses anteriores a la fecha de alta.

c) Normalización de la abstinencia, entendida como un "valor a preservar", integrado en la vida del paciente, sin que sea percibido como un lastre conductual, emocional o relacional.

d) Monóxido de Carbono (CO) igual o menor a 5 ppm. 
Se ha creado una variable denominada RET "Retención a los 12 meses" que toma el valor "SI", si el tipo de alta es ALTA TERAPEUTICA, cumpliendo los criterios señalados y "NO" si el paciente abandona el programa por recaída sin remisión parcial, denominándose ABANDONO. Cuando es alta es VOLUNTARIA la variable RET toma el valor "SI" si se constata un periodo de abstinencia continuada a los 12 meses, fecha de alta.

La retención se refiere a la abstinencia continuada en el programa, considerándose la posibilidad de alta terapéutica en caso de que se haya constatado la abstinencia continuada 6 meses antes de la fecha de ALTA, en ausencia de recaída franca (más de una semana de consumos diarios de cigarrillos).

Así pues la retención indica "alta terapéutica" a los 12 meses del programa.

El $77,30 \%$ de los pacientes se mantienen abstinentes al año de tratamiento, habiendo sido dados de alta.

\section{Retención a los 12 meses por sexo}

La siguiente tabla presenta la distribución, total y por sexo, de la variable retención.
Podemos ver como el porcentaje de pacientes retenidos a los 12 meses es similar entre hombres y mujeres (78.1\% y $76.6 \%$, respectivamente) no detectándose que la diferencia sea significativa, según el test de la Chi cuadrado. El gráfico siguiente muestra dicha distribución. La figura siguiente muestra dicha distribución:

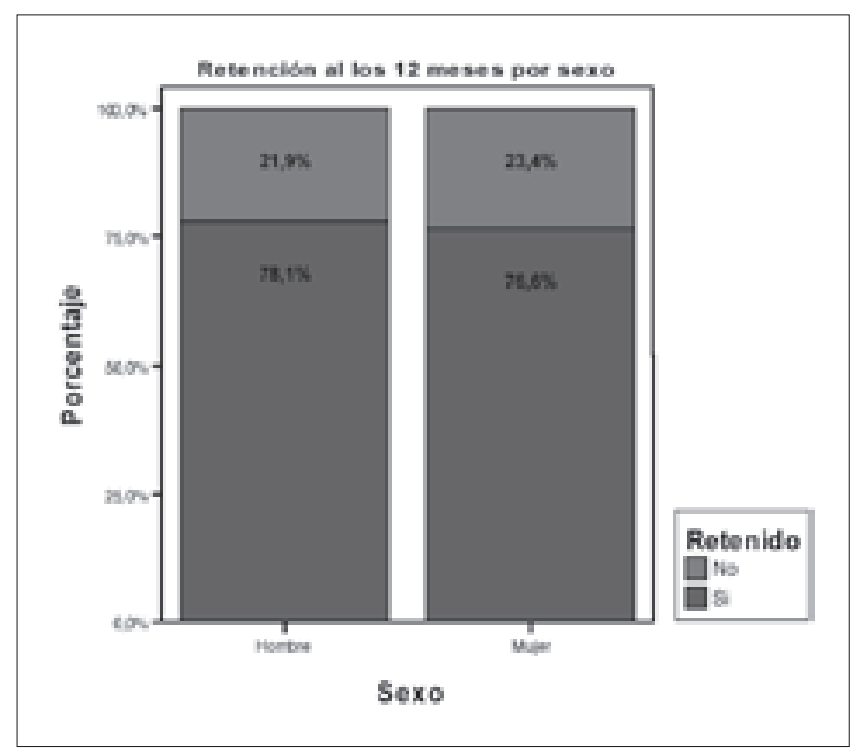

FIGURA 7. Retención porcentual a los 12 meses de tratamiento por sexo.

TABLA 4. Retención en el programa a los 12 meses de tratamiento por sexo.

\begin{tabular}{|c|c|c|c|c|c|c|}
\hline & \multirow{2}{*}{\multicolumn{2}{|c|}{ TOTAL }} & \multicolumn{4}{|c|}{ Sexo del paciente } \\
\hline & & & \multicolumn{2}{|c|}{ Hombre } & \multicolumn{2}{|c|}{ Mujer } \\
\hline & Casos & $\%$ & Casos & $\%$ & Casos & $\%$ \\
\hline $\begin{array}{l}\text { TOTAL } \\
\text { No } \\
\text { Si }\end{array}$ & $\begin{array}{r}128 \\
29 \\
99\end{array}$ & $\begin{array}{r}100,0 \% \\
22,7 \% \\
77,3 \%\end{array}$ & $\begin{array}{l}64 \\
14 \\
50\end{array}$ & $\begin{array}{r}100,0 \% \\
21,9 \% \\
78,1 \%\end{array}$ & $\begin{array}{l}64 \\
15 \\
49\end{array}$ & $\begin{array}{r}100,0 \% \\
23,4 \% \\
76,6 \%\end{array}$ \\
\hline
\end{tabular}

Después de la fecha de "alta", se realiza un seguimiento de la evolución de los pacientes. El segundo año se establecen 3 encuentros, y el tercero, uno, con el fin de conocer la continuidad o no de la abstinencia. En la actualidad estamos recogiendo esta información, si bien es progresiva en el tiempo, pues los pacientes no han sido reclutados de una sola vez.

\section{CONCLUSIONES}

1) La alta retención conseguida mediante el modelo terapéutico propuesto, nos sugiere considerar la relación con la duración del programa y la intensidad de la intervención (referida a número de sesiones y modalidad de tratamiento psicológico) como positiva.

2) Pensamos que este tipo de programa de abordaje clínico de la dependencia a nicotina no debe ser considerado de primera elección para todos los fumadores, puesto que un buen número de pacientes con dependencia a nicotina son capaces de dejar de fumar con intervenciones mínimas, con tratamientos farmacológicos y consejo médico, o bien con tratamientos psicológicos de menos intensidad y duración.

Sin embargo, teniendo en cuenta que los resultados más positivos de abstinencia continuada al año de inicio de la cesación, oscilan entre el $20 \%$ y el $45 \%{ }^{90}$, 91, 92, 93, 94, creemos oportuno, a la vista de los resultados obtenidos, ponderar la posibilidad de aumentar 
la intensidad de tratamiento psicológico en un determinado grupo de pacientes fumadores, resistentes a otros tipos de intervención.

3) Un buen número de fumadores expresa posibles dificultades abstinenciales en las 3 primeras sesiones de tratamiento, que se relacionan con problemas emocionales y/o relacionales que parecen limitar sus expectativas de cesación con éxito. Es por ello que consideramos oportuno complementar la exploración diagnóstica con herramientas como el genograma, de sencilla utilización, pero muy eficaz a la hora de permitir entender aspectos de dificultad predictores de recaída a corto y medio plazo. Este hecho, así como la constatación de recaída a largo plazo relacionada con factores de estrés negativo, justifican nuestras reflexiones sobre la posibilidad de aumentar el grado de complejidad del tratamiento de la dependencia a nicotina, si bien no existen referencias científicas sobre intervenciones de carácter sistémico- relacional en el tratamiento del tabaquismo.

4) El estudio tiene las limitaciones propias de un análisis descriptivo de casos, no pudiendo establecer criterios de eficacia al no haber realizado un diseño con grupo control. Serán necesarios estudios controlados para conocer con más profundidad, qué variables son las que determinan el alto porcentaje de abstinencias continuadas obtenidas al año de comienzo de la cesación.

5) Los resultados indican que la terapia sustitutiva combinada junto con un modelo de tratamiento psicoterapéutico con enfoque sistémico relacional, parece aumentar notablemente el porcentaje de éxito, si bien en la actualidad estamos recogiendo los datos de seguimiento de abstinencia al segundo y tercer año de finalizado el programa.

6) Desde nuestra experiencia clínica consideramos adecuado abrir el abanico de herramientas terapéuticas que permitan entender las dificultades de los pacientes fumadores. El proceso de tratamiento psicológico puede tener una gran riqueza de posibles intervenciones que, al menos, pueden ser consideradas, aunque no sean sistemáticamente utilizadas, pues pueden favorecer el aumento de la probabilidad de desvinculación satisfactoria del tóxico, en simetría con la redefinición de otros conflictos latentes en los que se inserta de manera poderosa la conducta adictiva. Si bien un buen número de fumadores es capaz de desvincularse con facilidad de la dependencia a nicotina, existe un número de personas en las que es necesario aceptar la complejidad de su dependencia, buscando alternativas terapéuticas que reconduzcan la angustia, la percepción de imposibilidad para el cambio. Desde la óptica sistémico-relacional, pueden redefinirse algunas de las dificultades más notables expresadas por los fumadores con alta sensación de dependencia que permitan la activación del cambio.

\section{REFERENCIAS}

1. Agencia de Evaluación de Tecnologías Sanitarias. Instituto de Salud Carlos III. MSC. Evaluación de la eficacia, efectividad y coste-efectividad de los distintos abordajes terapéuticos para dejar de fumar." Madrid: AETS- Instituto de Salud Carlos III, septiembre 2003.

2. Ezzati M, López AD. Estimates of global mortality attributable to smoking in 2000. Lancet 2003; 362: 847-852.

3. Banegas JR, Díez L, Rodríguez-Artalejo F, González J, Graciano A, Villar F. Mortalidad atribuible al tabaquismo en España en 1998. Med Clin 2001; 117:692-694.

4. Montes A, Pérez M, Gestal JJ. Impacto del tabaquismo sobre la mortalidad en España. Adicciones 2004, vol 16, Supl. 2.

5. Ministerio de Sanidad y Consumo. Encuesta Nacional de Salud 2001. Madrid: MSC (disponible URL: http:// www. msc.es/salud/epidemiologia /home.htm (mayo 2003).

6. Ministerio de Sanidad y Consumo. Encuesta Nacional de salud 2003. Madrid: MSC. INE,2005.

7. Ministerio de sanidad y Consumo. Encuesta Nacional de Salud, 1987. Madrid: MSC; 1989.

8. World Health Organization. International Statistical Classification of Diseases and related Health problems, 10th revision. Geneva: WHO; 1992.

9. American Psychiatric Association (2000). Diagnostic and Statistical Manual of Mental Disorders, $4^{\text {th }}$ edition, revised text. Washington D.F: Americam Psychiatric Association (trad. cast.: Barcelona, Masson, 2002).

10. Maddux, J.F. y Desmond, D.P. Addiction or dependence? Addiction 2000, 95: 661-665.

11. Marina R. Picciotto and William A. Corrigall. Neuronal Systems Underlying Behaviors Related to Nicotine Addiction: Neural Circuits and Molecular Genetics. The Journal of Neuroscience, 2002; 22:3338-3341.

12. Berrendero F, Mendizabal V, Robledo P, Galeote L, Bilkei-Gorzo A, Zimmer A, Maldonado R. Induced Antinociception, Rewarding Effects, and Physical Dependence Are Decreased in Mice Lacking the Preproenkephalin Gene. The Journal of Neuroscience, 2005; 25:1103-1112.

13. Fuentes-Pila JM, Cortijo C. Tabaquismo: hábito frente a adicción. El Farmacéutico, 2000. 250.

14. Royce, J.R. y Powell, A.: Theory of personality and individual differences: Factors, systems and processes. Englewood Cliffs, NJ,1983: Prentice-hall.

15. González-Pienda, J.A., Núñez, J.C. y Valle, A.: Influencia de los procesos de comparación interna/externa sobre la formación del autoconcepto y su relación con el rendimiento académico. Revista de Psicología General y Aplicada, 1992; 45: 73-82.

16. Gardner, H.: Inteligencias múltiples. La teoría en la práctica. Barcelona: Paidós, 1995.

17. Gallagher, J.J: Teaching and learning: New models. Annual Review of Psychology, 1994; 45: 171-195. 
18. Royal Collage of Physicians. Nicotine addiction in Britain. A report of the Tobacco Advisory Group of the Royal Collage of Physicians. London: Royal College of Physicians; 2000.

19. Kaplan H, Sadock BJ. Compendio de Psiquiatría. Ed. Masson. Barcelona.1991.

20. Ministerio de Sanidad y Consumo. Encuesta Nacional de Salud de España, 1997. Madrid: MSC; 1999.

21. Becoña $E$, Vázquez F. Dejar de fumar como proceso: implicaciones asistenciales. En: Libro blanco sobre tabaquismo en España. Barcelona: Glosa; 1998.

22. Jiménez $C$, Barrueco $M$, Solano S., Torrecilla $M$, Domínguez F, Díaz- Maroto JL et al. Recomendaciones en el abordaje diagnóstico y terapéutico del tabaquismo. Documento de Consenso Prev Tab, 2002; 4:147-155.

23. Becoña E. Evaluación de la conducta de fumar. En JL Graña (Ed.) Conductas adictivas. Teoría, evaluación y tratamiento. Madrid, Debate, 1994.

24. Doherty K, Kinnunen T, Militello FS, Garvey AJ. Urges to smoke during the first month of abstinence: relationship to relapse and predictors. Psychopharmacology, 1995; 119:171-178.

25. Tiffany ST, Drobes DJ. The development and initial validation of a questionnaire on smoking urges. British J Addiction, 1991; 86:1467-76.

26. Condiotte MM, Lichtenstein E. Self- efficacy and relapse in smoking cessation programs. Journal of Consulting and Clinical Psychology, 1981; 49:648-658.

27. DiClemente CC. Self- efficacy and smoking cessation maintenance. Cognitive Therapy and Research, 1981; 5: 175-187.

28. Brandon TH, Baker TB. The smoking Consequences Questionnaire: The subjetive expected utility of smoking in college students. Psychological Assessment, 1991; 3: 484-491

29. Fageström KO. Measuring degree of physical dependence on tobacco smoking with references to individualization of treatment. Addictive Behaviors, 1978; 3:235-241.

30. Fageström KO, Schneider NG. Measuring nicotine dependence: A review of the Fageström Tolerance Questionnaire. Journal of Behavioral Medicine, 1989; 12:159-182.

31. Heatherton, T.F., Kozlowski, L.T., Frecker, R.C. y Fagerström, K.O. The Fagerström Test for Nicotine Dependence: A revision of the Fagerström Tolerance Questionnaire. British Journal of Addictions, 1991; 86:1119-1127.

32. Fageström KO, Schneider NG. Measuring nicotine dependence: A review of the Fageström Tolerance Questionnaire. Journal of Behavioral Medicine, 1989; 12: 159-182.

33. Fiore MC, Novotny T, Pierce J, Giovino G, Hatziandreu E, Newcomb P, Surawicz T, Davis R. Methods used to quit smoking in the united States: Do cessation programs help? Journal of the American Medical Association, 1990; 263: 2760-68.

34. Prochaska JO, DiClemente CC. Stages and Proceses of self-change of smoking: Toward an integrative odel of change. Journal of Consulting and Clinical Psychology, 1983; 51:390-395.

35. Prochaska JO, Prochaska JM. Modelo transteórico de cambio para conductas adictivas. Recaída y Prevención de Recaídas. Ed. Neurociencias. CITRAN, Barcelona, 1993.

36. DiClemente CC. Motivational interviewing and the stages of change. In: Miller WR and Rollnick S. eds. Motivational Interviewing: Preparing People To Change Addictive Behaviors. New York, Guilford Press, 1993.

37. DiClemente CC, Prochaska JO. Self-change and therapy change of smoking behavior: A comparison of processes of change of cessation and maintenancce. Addictive Behaviors, 1982; 7:133-142.

38. Marlatt GA. LA prevención de recaídas en las conductas adictivas. Recaída y Prevención de recaídas. Ed. Neurociencias. CITRAN. Barcelona, 1993.

39. Bandura A. Social Foundations of Thought and Action: A Social Cognitive Theory. Englewood Cliffs, NJ: PrenticeHall; 1986.

40. Martín F, Corral P. Tratamiento farmacológico del tabaquismo. Trastornos Adictivos, 2004; 6:103-112.

41. Jiménez C, de Granda JI, Solano S, Mayayo ML. Tratamiento del Tabaquismo. ¿Qué hay de nuevo? Revista de Patología Respiratoria, 2004; 7:70-72.

42. King A, de Wit H, Riley R, Cao D, Niaura R, Hatsukami D. Efficacy of naltrexona in smoking cessation: A preliminary study and an examination of sex differences. Nicotine Tobacco Research, 2006; 8 : 671682.

43. Schwartz, J.L. Review and evaluation of smoking cessation methods The United States and Canada, 1978-1985. Washington, DC:U.S. Department of Health and Human Services, 1987.

44. Becoña, E. El tratamiento psicológico de la adicción a nicotina. Papeles del psicólogo, 2003; 85: 48-69.

45. Fiore, M.C., Bailey W.C., Cohen S.J., Dorfman S.F., Goldstein M.G., Gritz E.R., et al. Treating tobacco use and dependence. Rockville, MD; U.S. Department of Health and Human Services, Public Health Service, 2000.

46. Niaura R. y Abrams, D.B. Smoking cessation: Progress, priorities and prospectus. Journal of Consulting and Clinical Psychology, 2002; 70, 494-509.

47. Becoña E. Tratamiento del tabaquismo. Situación actual y perspectivas futuras. Adicciones, 2000; 12: 77-85.

48. Lancaster T, Stead LF. Individual behavioural counselling for smoking cessation (Cochrane Review). En: The Cochrane Library, Issue 2. Oxford. Update Sofware; 2003.

49. Stead LF, Lancaster T. Group behaviour therapy programmes for smoking cessation (Cochrane Review). 
En: The Cochrane Library, Issue 2. Oxford: Update Software; 2003.

50. Hajek P, Stead LF. Aversive smoking for smoking cessation (Cochrane Review). En: The Cochrane Library, Issue 2. Oxford: Update Software; 2003.

51. Secker- Walter RH, Gnich W,Platt S, Lancaster T. Community interventions for reducing smoking among adults. The Cochrane Library, Issue 2. Oxford: Update Software; 2003.

52. Whitaker C.A., Bumberry W.M. Danzando con la familia. Un enfoque simbólico-experiencial. Ed. Paidós. Barcelona, 1991.

53. Whitaker C., Ryan M.O.(c): Meditaciones nocturnas de un terapeuta familiar. Ed. Paidós. Barcelona, 1992.

54. Fuentes-Pila JM, Calatayud P, López E, Castañeda B. La entrevista motivacional: llave del proceso de cambio en la dependencia nicotín-tabáquica. Trastornos Adictivos, 2005; 7:153-65.

55. Fuentes-Pila JM, López E., Castañeda B, Calatayud P. Diagnóstico del gran fumador. Dificultades relacionadas con el concepto hábito de fumar. Prevención de Tabaquismo, 2004; 6(l):164.

56. Volkow ND, Ting-Kai L. Drug addiction: the neurobiology of behaviour gone awry: Nature Neuroscience Reviews, 2004; 5:963-970.

57. Bowen M. De la familia al individuo. La diferenciación del sí mismo en el sistema familiar, Barcelona, Paidós, 1993.

58. McGoldrick M. "Some data on death and cancer in schizofrenic families". Informe presentado en el Presymposium Conference of Georgetown Symposium, Washington DC, 1977.

59. Lieberman S. Transgenerational Family Therapy. Londres. Croom Helm, 1979.

60. Fuentes-Pila JM, López E, Castañeda B, Calatayud P. El Genograma. Uso clínico en el tratamiento del tabaquismo. Prev Tab 2005; 7:97-108.

61. McGoldrick M, Gerson R. Genogramas en la evaluación familiar. Gedisa, Buenos Aires; Barcelona, 1993.

62. Koob G, Le Moal M. Drug Abuse: Hedonic homeostatic dysregulation. Science 1997; 278: 52-58.

63. Gossop M, Powell J, Grey S, Hajek P. What do opiate addicts and cigarette smokers mean by "craving"? A pilot study. Drug Alcohol Depend 1990; 26-85-7.

64. Nichols M.P., Minuchin S. La recuperación de la familia. Relatos de esperanza y renovación. Editorial Paidós. Barcelona, 1994.

65. Minuchin S. Técnicas de Terapia Familiar. Ed. Paidós, Buenos Aires, 1984.

66. Minuchin S. Familias y Terapia Familiar. Ed. Gedisa, Barcelona, 1979.

67. Cade B., O’Hanlon. Guía breve de terapia breve. Barcelona, Paidos, 1995.

68. Haley H. Las tácticas de poder de Jesucristo y otros ensayos. Barcelona, Paidos 1991.
69. Haley J. Tratamiento de la familia. Ed. Toray, Barcelona, 1974.

70. Haley J. Terapia no convencional. Las técnicas psiquiátricas de Milton Erickson. Ed. Amorrortu, Buenos Aires, 1980.

71. Bertalanffy L. Teoría General de los Sistemas. Ed. Fondo de Cultura Económica, México. 1976.

72. Watzlawick P, Beavin J, Jackson D. Teoría de la Comunicación Humana. Ed. Herder, Barcelona, 1981.

73. De Shazer, S. Putting Differences to Work. Nueva York, Norton, 1991.

74. De Shazer, S. En un origen las palabras eran mágicas. Ed. Gedisa. Barcelona, 1999.

75. Kim Berg I, Miller S. Trabajando con el problema del alcohol. Orientaciones y sugerencias para la terapia breve de familia. p.33. Ed. Gedisa. Barcelona, 2002.

76. De Shazer, S. Claves en psicoterapia breve. Una teoría de la solución. Ed. Gedisa, Barcelona, 1997.

77. Friedman S. El Nuevo lenguaje del Cambio. La colaboración constructiva en psicoterapia. Ed. Gedisa. Barcelona, 2001.

78. Nardone G., Watzlawick P. El arte del cambio, Herder, Barcelona, 1992.

79. Nardone G, Salvini A. El diálogo estratégico. Ed. RBA, Barcelona, 2006.

80. Watzlawick P. El lenguaje del cambio, Herder, Barcelona, 1980.

81. H.Maturana and F.Varela, De Máquinas y Seres Vivos: Una teoría sobre la organización biológica, Editorial Universitaria, Santiago, 1973, 121 pp. New revised edition with a Preface, 1995.

82. H.Maturana and F. Varela, El Arbol del Conocimiento: Las bases biológicas del entendimiento humano, Editorial Universitaria, Santiago, 1985; Editorial Debate, Madrid, 1988.

83. Gergen K.J. El yo saturado: dilemas de identidad en el mundo contemporáneo, Barcelona, Paidos, 1997.

84. Gergen K.J. An invitation to Social Construction, Londres, Sage, 1999.

85. White M, Epston D. Medios narrativos para fines terapéuticos. Barcelona, Paidós, 1993.

86. White $\mathrm{M}$. El enfoque narrativo en la experiencia de los terapeutas. Ed. Gedisa, Barcelona, 2002.

87. White M. Reescribir la vida. Entrevistas y ensayos. Ed. Gedisa. Barcelona, 2002.

88. Gergen K, Gergen M. Narrative and the self as relationship. In L. Borkowitz (ed). Advances in experimental social psychology. NY, Academic Press, 1986.

89. Shoeetter, J. Circular interviewing: A multifaceted clinical tool. In D. Campbell \& R. Draper (eds), applications of systemic therapy: The Milan approach. London: Grune \& Strarion, 1985.

90. COMMIT Research Group: Community intervention trial for smoking cessation: changes in adult cigarette 
smoking prevalence. Am J Public Health 1995; 85:193200.

91. Corral C, Pascual M. Intervención sobre el tabaquismo en atención primaria. Estudio de cuatro modalidades de actuación. Atención Primaria, 1992; 9:287-292.

92. García Carmona T, Milán Santos I, Ferreiro Álvarez MJ. Evaluación de los resultados obtenidos durante el primer año en la consulta de tabaquismo de la Clínica Puerta de Hierro. Prevención de Tabaquismo, 2001; 3:58-63.
93. Jiménez Mas F, Gotor Ciller MI, Mateos Ramos A, Almar Marqués E, Jiménez García M. Tratamiento multicomponente del tabaquismo en pacientes con potencial ejemplificante por su profesión. Prevención del Tabaquismo, 2001; 3:132-137.

94. Jiménez Ruiz CA, Alonso S, Almonacid C, Cisneros C, Perelló O, Barrueco $\mathrm{M}$ et al. Tratamiento combinado: chicles más parches de nicotina en fumadores con alta dependencia. Rev Patol Respir 2000; 1:5-8. 
